Georgian Mathematical Journal

Volume 14 (2007), Number 4, 775-792

\title{
EXISTENCE OF MULTIPLE POSITIVE SOLUTIONS FOR EVEN ORDER MULTI-POINT BOUNDARY VALUE PROBLEMS
}

\author{
YOUYU WANG AND WEIGAO GE
}

\begin{abstract}
In this paper, we consider the existence of multiple positive solutions for the $2 n$th order $m$-point boundary value problem:

$$
\begin{cases}x^{(2 n)}(t)=f\left(t, x(t), x^{\prime \prime}(t), \cdots, x^{(2(n-1))}(t)\right), & 0 \leq t \leq 1, \\ x^{(2 i)}(0)-a_{i} x^{(2 i+1)}(0)=\sum_{j=1}^{m-2} \alpha_{i j} x^{(2 i)}\left(\xi_{j}\right), & \\ x^{(2 i)}(1)+b_{i} x^{(2 i+1)}(1)=\sum_{j=1}^{m-2} \beta_{i j} x^{(2 i)}\left(\xi_{j}\right), & 0 \leq i \leq n-1,\end{cases}
$$

where $\alpha_{i j}, \beta_{i j}, a_{i}, b_{i} \in[0, \infty)(0 \leq i \leq n-1,1 \leq j \leq m-2), \sum_{j=1}^{m-2} \alpha_{i j}, \sum_{j=1}^{m-2} \beta_{i j} \in$ $(0,1), 0<\xi_{1}<\xi_{2}<\cdots<\xi_{m-2}<1$. Using the Leggett-Williams fixed point theorem, we provide sufficient conditions for the existence of at least three positive solutions to the above boundary value problem. The associated Green's function for the above problem is also given.
\end{abstract}

2000 Mathematics Subject Classification: 34B10, 34B15.

Key words and phrases: Higher order $m$-point boundary value problem, Leggett-Williams fixed point theorem, Green's function, positive solution.

\section{INTRODUCTION}

Multi-point boundary value problems for ordinary differential equations arise in a variety of areas of applied mathematics and physics. Linear and nonlinear second order multi-point boundary value problems have also been studied by several authors. We refer the reader to [2]-[8] and the references therein. Davis et al. [9] and Davis et al. [10] studied the 2nth order Lidstone BVP

$$
\left\{\begin{array}{l}
x^{(2 n)}=f\left(x(t), x^{\prime \prime}(t), \ldots, x^{(2(n-1))}(t)\right), \quad t \in[0,1] \\
x^{(2 i)}(0)=x^{(2 i)}(1)=0, \quad 0 \leq i \leq n-1
\end{array}\right.
$$

where $(-1)^{n} f: R^{n} \rightarrow[0, \infty)$ is continuous. They obtained the existence of three symmetric positive solutions of the BVP (1).

Recently, Y. Guo et al. [11] studied the following $2 n$th order BVP

$$
\left\{\begin{array}{l}
x^{(2 n)}(t)=f\left(t, x(t), x^{\prime \prime}(t), \ldots, x^{(2(n-1))}(t)\right), \quad 0 \leq t \leq 1 \\
x^{(2 i)}(0)-\beta_{i} x^{(2 i+1)}(0)=0, x^{(2 i)}(1)=\sum_{j=1}^{m-2} k_{i j} y^{(2 i)}\left(\xi_{j}\right), \quad 0 \leq i \leq n-1 .
\end{array}\right.
$$


They established the existence of at least three positive solution for the above BVP.

Y. Guo et al. [12] also studied the $2 n$th order BVP

$$
\left\{\begin{array}{l}
x^{(2 n)}(t)=f\left(t, x(t), x^{\prime \prime}(t), \ldots, x^{(2(n-1))}(t)\right), \quad 0 \leq t \leq 1 \\
x^{(2 i)}(0)=0, x^{(2 i)}(1)=\sum_{j=1}^{m-2} k_{i j} y^{(2 i)}\left(\xi_{j}\right), \quad 0 \leq i \leq n-1 .
\end{array}\right.
$$

By using the Leggett-Williams fixed point theorem, they got at least three positive solutions for the BVP (3). Motivated by the results of [9] and [12], in this paper we study the existence of multiple positive solutions for the $2 n$th order $m$-point boundary value problem

$$
\left\{\begin{array}{l}
x^{(2 n)}(t)=f\left(t, x(t), x^{\prime \prime}(t), \ldots, x^{(2(n-1))}(t)\right), \quad 0 \leq t \leq 1, \\
x^{(2 i)}(0)-a_{i} x^{(2 i+1)}(0)=\sum_{j=1}^{m-2} \alpha_{i j} x^{(2 i)}\left(\xi_{j}\right), \\
x^{(2 i)}(1)+b_{i} x^{(2 i+1)}(1)=\sum_{j=1}^{m-2} \beta_{i j} x^{(2 i)}\left(\xi_{j}\right), \quad 0 \leq i \leq n-1 .
\end{array}\right.
$$

To the best of our knowledge, the existence results for positive solutions of the above boundary value problem have not been studied previously.

Throughout the paper, we assume that the following conditions are satisfied:

$\left(H_{1}\right) \alpha_{i j}, \beta_{i j}, a_{i}, b_{i}(0 \leq i \leq n-1,1 \leq j \leq m-2) \in[0, \infty), \sum_{j=1}^{m-2} \alpha_{i j}, \sum_{j=1}^{m-2} \beta_{i j} \in$ $(0,1), 0<\xi_{1}<\xi_{2}<\ldots<\xi_{m-2}<1$;

$\left(H_{2}\right)(-1)^{n} f:[0,1] \times R^{n} \rightarrow[0, \infty)$ is continuous.

\section{Preliminaries}

Our main results will depend on the Leggett-Williams fixed-point theorem. For convenience, we present here the necessary definitions from the theory of cones in Banach spaces.

Definition 2.1. Let $E$ be a real Banach space. A nonempty convex closed set $P \subset E$ is said to be a cone provided that

(i) $a u \in P$ for all $u \in P$ and all $a \geq 0$ and

(ii) $u,-u \in P$ implies $u=0$.

Note that every cone $P \subset E$ induces an ordering in $E$ given by $x \leq y$ if $y-x \in P$.

Definition 2.2. A map $\alpha$ is said to be a nonnegative continuous concave functional on a cone $P$ of a real Banach space $E$ provided that $\alpha: P \rightarrow[0, \infty)$ is continuous and

$$
\alpha(t x+(1-t) y) \geq t \alpha(x)+(1-t) \alpha(y)
$$

for all $x, y \in P$ and $0 \leq t \leq 1$.

Similarly, we say that a map $\beta$ is a nonnegative continuous convex functional on a cone $P$ of a real Banach space $E$ provided that $\beta: P \rightarrow[0, \infty)$ is continuous 
and

$$
\beta(t x+(1-t) y) \leq t \beta(x)+(1-t) \beta(y)
$$

for all $x, y \in P$ and $0 \leq t \leq 1$.

Definition 2.3. An operator is called completely continuous if it is continuous and maps bounded sets into pre-compact sets.

For positive real numbers $a, b$, we define the following convex sets:

$$
\begin{gathered}
P_{r}=\{x \in P \mid\|x\|<r\}, \\
P(\alpha, a, b)=\{x \in P \mid a \leq \alpha(x), \quad\|x\| \leq b\} .
\end{gathered}
$$

Theorem 2.1 ([1], Leggett-Williams Fixed Point Theorem). Let $A: \bar{P}_{c} \rightarrow$ $\bar{P}_{c}$ be a completely continuous operator and let $\alpha$ be a nonnegative continuous concave function on $P$ such that $\alpha(x) \leq\|x\|$ for all $x \in \bar{P}_{c}$. Suppose there exists $0<a<b<d \leq c$ such that

(C1) $\{x \in P(\alpha, b, d) \mid \alpha(x)>b\} \neq \varnothing \quad$ and $\quad \alpha(A x)>b$ for $x \in P(\alpha, b, d)$,

(C2) $\|A x\|<a$ for $\|x\| \leq a$ and

(C3) $\alpha(A x)>b$ for $x \in P(\alpha, b, c)$ with $\|A x\|>d$.

Then $A$ has at least three fixed points $x_{1}, x_{2}$ and $x_{3}$ such that $\left\|x_{1}\right\|<a, b<$ $\alpha\left(x_{2}\right)$, and $\left\|x_{3}\right\|>a$ with $\alpha\left(x_{3}\right)<b$.

\section{Multiple Positive Solutions of (4)}

In order to apply Theorem 2.1, we must define an appropriate operator on a Banach space. We first consider the unique solution of the following second order boundary value problem.

Lemma 3.1. Suppose $\left(1-\sum_{i=1}^{m-2} \beta_{i}\right)\left(a+\sum_{i=1}^{m-2} \alpha_{i} \xi_{i}\right)+\left(1-\sum_{i=1}^{m-2} \alpha_{i}\right)(b+1-$ $\left.\sum_{i=1}^{m-2} \beta_{i} \xi_{i}\right) \neq 0 . \quad$ If $f(t) \in C[0,1]$. Then the problem

$$
\left\{\begin{array}{l}
x^{\prime \prime}(t)+f(t)=0, \quad 0 \leq t \leq 1, \\
x(0)-a x^{\prime}(0)=\sum_{i=1}^{m-2} \alpha_{i} x\left(\xi_{i}\right), \quad x(1)+b x^{\prime}(1)=\sum_{i=1}^{m-2} \beta_{i} x\left(\xi_{i}\right),
\end{array}\right.
$$

has a unique solution

$$
x(t)=-\int_{0}^{t}(t-s) f(s) d s+A t+B,
$$

where

$$
\begin{aligned}
A= & \frac{1}{M}\left[\left(1-\sum_{i=1}^{m-2} \beta_{i}\right)\left(\sum_{i=1}^{m-2} \alpha_{i} \int_{0}^{\xi_{i}}\left(\xi_{i}-s\right) f(s) d s\right)\right. \\
& +\left(1-\sum_{i=1}^{m-2} \alpha_{i}\right) \int_{0}^{1}(b+1-s) f(s) d s
\end{aligned}
$$




$$
\begin{aligned}
& \left.-\left(1-\sum_{i=1}^{m-2} \alpha_{i}\right)\left(\sum_{i=1}^{m-2} \beta_{i} \int_{0}^{\xi_{i}}\left(\xi_{i}-s\right) f(s) d s\right)\right], \\
B= & \frac{1}{M}\left[\left(a+\sum_{i=1}^{m-2} \alpha_{i} \xi_{i}\right) \int_{0}^{1}(b+1-s) f(s) d s\right. \\
& -\left(a+\sum_{i=1}^{m-2} \alpha_{i} \xi_{i}\right)\left(\sum_{i=1}^{m-2} \beta_{i} \int_{0}^{\xi_{i}}\left(\xi_{i}-s\right) f(s) d s\right) \\
& \left.-\left(b+1-\sum_{i=1}^{m-2} \beta_{i} \xi_{i}\right)\left(\sum_{i=1}^{m-2} \alpha_{i} \int_{0}^{\xi_{i}}\left(\xi_{i}-s\right) f(s) d s\right)\right], \\
M= & \left(1-\sum_{i=1}^{m-2} \beta_{i}\right)\left(a+\sum_{i=1}^{m-2} \alpha_{i} \xi_{i}\right)+\left(1-\sum_{i=1}^{m-2} \alpha_{i}\right)\left(b+1-\sum_{i=1}^{m-2} \beta_{i} \xi_{i}\right) .
\end{aligned}
$$

Proof. Assume that

$$
x(t)=-\int_{0}^{t}(t-s) f(s) d s+A t+B
$$

according to the boundary conditions, we have

$$
\begin{aligned}
-A\left(a+\sum_{i=1}^{m-2} \alpha_{i} \xi_{i}\right)+B\left(1-\sum_{i=1}^{m-2} \alpha_{i}\right) & =-\sum_{i=1}^{m-2} \alpha_{i} \int_{0}^{\xi_{i}}\left(\xi_{i}-s\right) f(s) d s \\
A\left(b+1-\sum_{i=1}^{m-2} \beta_{i} \xi_{i}\right)+B\left(1-\sum_{i=1}^{m-2} \beta_{i}\right)= & -\sum_{i=1}^{m-2} \beta_{i} \int_{0}^{\xi_{i}}\left(\xi_{i}-s\right) f(s) d s \\
& +\int_{0}^{1}(b+1-s) f(s) d s .
\end{aligned}
$$

Now it is easy to check our results.

Lemma 3.2. Let $M=\left(1-\sum_{i=1}^{m-2} \beta_{i}\right)\left(a+\sum_{i=1}^{m-2} \alpha_{i} \xi_{i}\right)+\left(1-\sum_{i=1}^{m-2} \alpha_{i}\right)(b+1-$ $\left.\sum_{i=1}^{m-2} \beta_{i} \xi_{i}\right) \neq 0 . \quad$ Then the Green's function for the boundary value problem

$$
\left\{\begin{array}{l}
-x^{\prime \prime}(t)=0, \quad 0 \leq t \leq 1, \\
x(0)-a x^{\prime}(0)=\sum_{i=1}^{m-2} \alpha_{i} x\left(\xi_{i}\right), \quad x(1)+b x^{\prime}(1)=\sum_{i=1}^{m-2} \beta_{i} x\left(\xi_{i}\right),
\end{array}\right.
$$


is given by

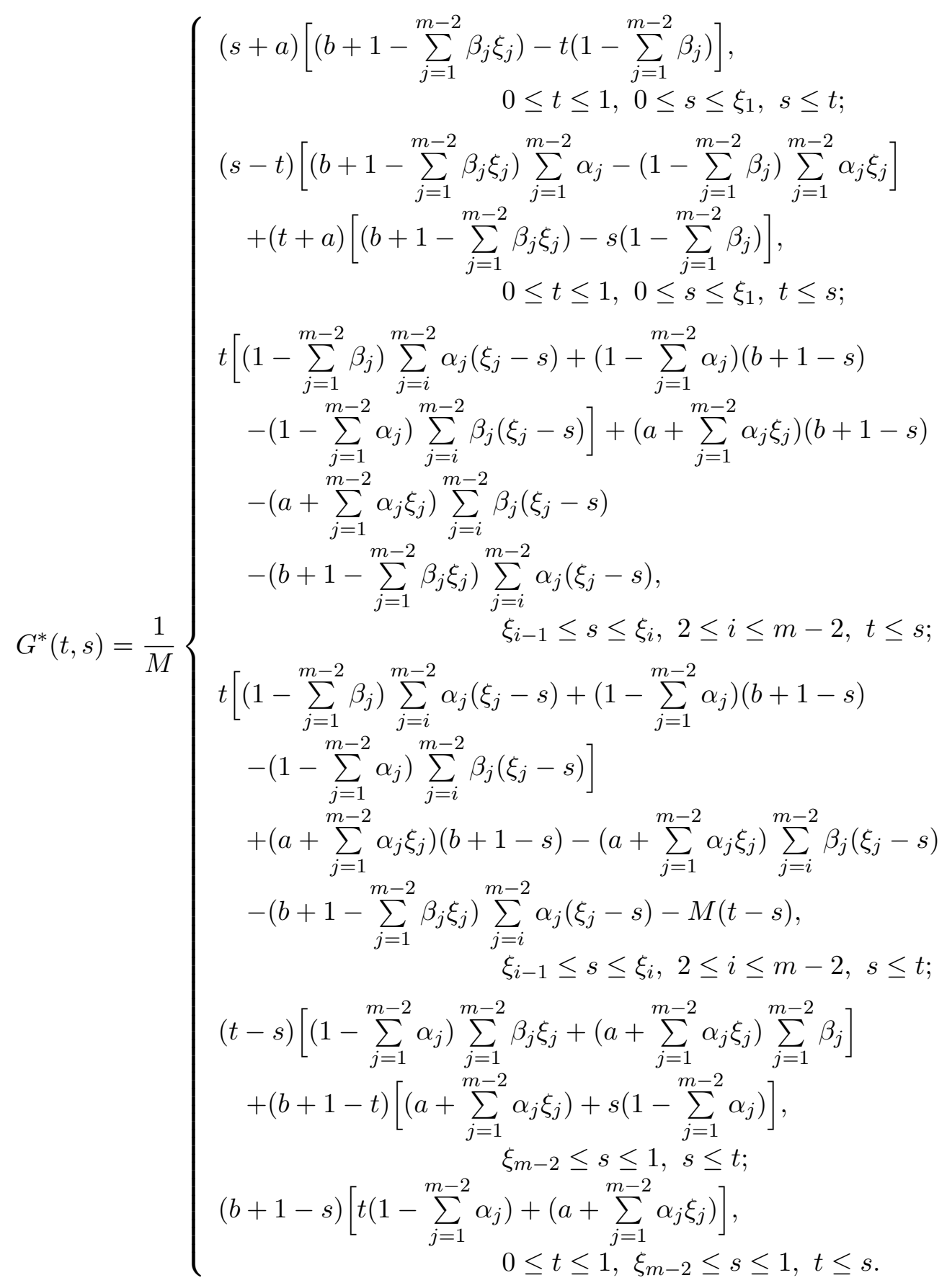

Lemma 3.3. Suppose $\alpha_{i}, \beta_{i}, a, b>0(i=1,2, \ldots, m-2), 0<\sum_{i=1}^{m-2} \alpha_{i}<1$, $0<\sum_{i=1}^{m-2} \beta_{i}<1$. Then

$$
G^{*}(t, s) \geq 0 \quad \text { for } \quad(t, s) \in[0,1] \times[0,1] .
$$

Proof. We consider only the following two cases. 
Case 1. We first check that if $\xi_{i-1} \leq s \leq \xi_{i}, 2 \leq i \leq m-2, t \leq s$, then

$$
\begin{aligned}
L_{3}:= & t\left(1-\sum_{j=1}^{m-2} \beta_{j}\right) \sum_{j=i}^{m-2} \alpha_{j}\left(\xi_{j}-s\right)+\left(1-\sum_{j=1}^{m-2} \alpha_{j}\right)(b+1-s) \\
& \left.-\left(1-\sum_{j=1}^{m-2} \alpha_{j}\right) \sum_{j=i}^{m-2} \beta_{j}\left(\xi_{j}-s\right)\right] \\
& +\left(a+\sum_{j=1}^{m-2} \alpha_{j} \xi_{j}\right)(b+1-s)-\left(a+\sum_{j=1}^{m-2} \alpha_{j} \xi_{j}\right) \sum_{j=i}^{m-2} \beta_{j}\left(\xi_{j}-s\right) \\
& -\left(b+1-\sum_{j=1}^{m-2} \beta_{j} \xi_{j}\right) \sum_{j=i}^{m-2} \alpha_{j}\left(\xi_{j}-s\right) \\
\geq & 0 .
\end{aligned}
$$

Indeed, for the first part of the above formula we have

$$
\begin{aligned}
L_{31}:= & t\left(1-\sum_{j=1}^{m-2} \beta_{j}\right) \sum_{j=i}^{m-2} \alpha_{j}\left(\xi_{j}-s\right)+\left(1-\sum_{j=1}^{m-2} \alpha_{j}\right)(b+1-s) \\
& \left.-\left(1-\sum_{j=1}^{m-2} \alpha_{j}\right) \sum_{j=i}^{m-2} \beta_{j}\left(\xi_{j}-s\right)\right] \\
= & t\left(1-\sum_{j=1}^{m-2} \beta_{j}\right) \sum_{j=i}^{m-2} \alpha_{j}\left(\xi_{j}-s\right)+\left(1-\sum_{j=1}^{m-2} \alpha_{j}\right)\left(1-\sum_{j=i}^{m-2} \beta_{j} \xi_{j}\right. \\
& \left.\left.-s\left(1-\sum_{j=i}^{m-2} \beta_{j}\right)\right)\right]+b t\left(1-\sum_{j=1}^{m-2} \alpha_{j}\right) \\
\geq & 0
\end{aligned}
$$

while

$$
\begin{aligned}
L_{32}:= & \left(a+\sum_{j=1}^{m-2} \alpha_{j} \xi_{j}\right)(b+1-s)-\left(a+\sum_{j=1}^{m-2} \alpha_{j} \xi_{j}\right) \sum_{j=i}^{m-2} \beta_{j}\left(\xi_{j}-s\right) \\
& -\left(b+1-\sum_{j=1}^{m-2} \beta_{j} \xi_{j}\right) \sum_{j=i}^{m-2} \alpha_{j}\left(\xi_{j}-s\right) \\
\geq & \left(a+\sum_{j=1}^{m-2} \alpha_{j} \xi_{j}\right)(1-s)-\left(a+\sum_{j=1}^{m-2} \alpha_{j} \xi_{j}\right) \sum_{j=i}^{m-2} \beta_{j}\left(\xi_{j}-s\right) \\
& -\left(1-\sum_{j=1}^{m-2} \beta_{j} \xi_{j}\right) \sum_{j=i}^{m-2} \alpha_{j}\left(\xi_{j}-s\right) \\
= & s\left(a+\sum_{j=1}^{m-2} \alpha_{j} \xi_{j}\right) \sum_{j=i}^{m-2} \beta_{j}\left(1-\xi_{j}\right)+a\left(1-\sum_{j=i}^{m-2} \beta_{j} \xi_{j}\right)(1-s)
\end{aligned}
$$




$$
\begin{aligned}
& +s\left(1-\sum_{j=1}^{m-2} \beta_{j} \xi_{j}\right) \sum_{j=i}^{m-2} \alpha_{j}\left(1-\xi_{j}\right) \\
& +\left[\left(1-\sum_{j=i}^{m-2} \beta_{j} \xi_{j}\right) \sum_{j=1}^{m-2} \alpha_{j} \xi_{j}-\left(1-\sum_{j=1}^{m-2} \beta_{j} \xi_{j}\right) \sum_{j=i}^{m-2} \alpha_{j} \xi_{j}\right](1-s) \\
& \geq 0 .
\end{aligned}
$$

Therefore, $L_{3}=L_{31}+L_{32} \geq 0$.

Case 2. Now we show that if $\xi_{i-1} \leq s \leq \xi_{i}, \quad 2 \leq i \leq m-2, \quad s \leq t$, then

$$
\begin{aligned}
L_{4}:= & t\left[\left(1-\sum_{j=1}^{m-2} \beta_{j}\right) \sum_{j=i}^{m-2} \alpha_{j}\left(\xi_{j}-s\right)+\left(1-\sum_{j=1}^{m-2} \alpha_{j}\right)(b+1-s)\right. \\
& \left.-\left(1-\sum_{j=1}^{m-2} \alpha_{j}\right) \sum_{j=i}^{m-2} \beta_{j}\left(\xi_{j}-s\right)\right] \\
& +\left(a+\sum_{j=1}^{m-2} \alpha_{j} \xi_{j}\right)(b+1-s)-\left(a+\sum_{j=1}^{m-2} \alpha_{j} \xi_{j}\right) \sum_{j=i}^{m-2} \beta_{j}\left(\xi_{j}-s\right) \\
& -\left(b+1-\sum_{j=1}^{m-2} \beta_{j} \xi_{j}\right) \sum_{j=i}^{m-2} \alpha_{j}\left(\xi_{j}-s\right)-M(t-s) \\
\geq & 0 .
\end{aligned}
$$

From the above discussion we get

$$
\begin{aligned}
L_{43}:= & \left(a+\sum_{j=1}^{m-2} \alpha_{j} \xi_{j}\right)(b+1-s)-\left(a+\sum_{j=1}^{m-2} \alpha_{j} \xi_{j}\right) \sum_{j=i}^{m-2} \beta_{j}\left(\xi_{j}-s\right) \\
& -\left(b+1-\sum_{j=1}^{m-2} \beta_{j} \xi_{j}\right) \sum_{j=i}^{m-2} \alpha_{j}\left(\xi_{j}-s\right) \\
= & L_{32} \\
\geq & a\left(1-\sum_{j=i}^{m-2} \beta_{j} \xi_{j}\right)(1-s)+\left[\left(1-\sum_{j=i}^{m-2} \beta_{j} \xi_{j}\right) \sum_{j=1}^{m-2} \alpha_{j} \xi_{j}\right. \\
& \left.-\left(1-\sum_{j=1}^{m-2} \beta_{j} \xi_{j}\right) \sum_{j=i}^{m-2} \alpha_{j} \xi_{j}\right](1-s) \\
\geq & a\left(1-\sum_{j=i}^{m-2} \beta_{j} \xi_{j}\right)(1-s)+\left(1-\sum_{j=1}^{m-2} \beta_{j} \xi_{j}\right) \sum_{j=1}^{i-1} \alpha_{j} \xi_{j}(1-s) \\
\geq & a\left(1-\sum_{j=i}^{m-2} \beta_{j} \xi_{j}\right)(t-s)+\left(1-\sum_{j=1}^{m-2} \beta_{j}\right) \sum_{j=1}^{i-1} \alpha_{j} \xi_{j}(t-s) \\
\geq & a\left(1-\sum_{j=1}^{m-2} \beta_{j}\right)(t-s)+\left(1-\sum_{j=1}^{m-2} \beta_{j}\right) \sum_{j=1}^{i-1} \alpha_{j} \xi_{j}(t-s) .
\end{aligned}
$$


Analogously, we also have

$$
\begin{aligned}
L_{42}:= & t\left[\left(1-\sum_{j=1}^{m-2} \alpha_{j}\right)(b+1-s)-\left(1-\sum_{j=1}^{m-2} \alpha_{j}\right) \sum_{j=i}^{m-2} \beta_{j}\left(\xi_{j}-s\right)\right] \\
= & b\left(1-\sum_{j=1}^{m-2} \alpha_{j}\right) t+t s\left(1-\sum_{j=1}^{m-2} \alpha_{j}\right) \sum_{j=i}^{m-2} \beta_{j}\left(1-\xi_{j}\right) \\
& +\left(1-\sum_{j=1}^{m-2} \alpha_{j}\right)\left(1-\sum_{j=i}^{m-2} \beta_{j} \xi_{j}\right)(t-t s) \\
\geq & b\left(1-\sum_{j=1}^{m-2} \alpha_{j}\right)(t-s)+\left(1-\sum_{j=1}^{m-2} \alpha_{j}\right)\left(1-\sum_{j=1}^{m-2} \beta_{j} \xi_{j}\right)(t-s) \\
= & \left(1-\sum_{j=1}^{m-2} \alpha_{j}\right)\left(b+1-\sum_{j=1}^{m-2} \beta_{j} \xi_{j}\right)(t-s),
\end{aligned}
$$

again,

$$
\begin{aligned}
& t\left(1-\sum_{j=1}^{m-2} \beta_{j}\right) \sum_{j=i}^{m-2} \alpha_{j}\left(\xi_{j}-s\right)-\left(1-\sum_{j=1}^{m-2} \beta_{j}\right) \sum_{j=1}^{m-2} \alpha_{j} \xi_{j}(t-s) \\
= & t\left(1-\sum_{j=1}^{m-2} \beta_{j}\right) \sum_{j=i}^{m-2} \alpha_{j}\left(\xi_{j}-s\right) \\
& -\left(1-\sum_{j=1}^{m-2} \beta_{j}\right) \sum_{j=i}^{m-2} \alpha_{j} \xi_{j}(t-s)-\left(1-\sum_{j=1}^{m-2} \beta_{j}\right) \sum_{j=1}^{i-1} \alpha_{j} \xi_{j}(t-s) \\
= & s\left(1-\sum_{j=1}^{m-2} \beta_{j}\right) \sum_{j=i}^{m-2} \alpha_{j}\left(\xi_{j}-t\right)-\left(1-\sum_{j=1}^{m-2} \beta_{j}\right) \sum_{j=1}^{i-1} \alpha_{j} \xi_{j}(t-s) \\
\geq & -\left(1-\sum_{j=1}^{m-2} \beta_{j}\right) \sum_{j=1}^{i-1} \alpha_{j} \xi_{j}(t-s) .
\end{aligned}
$$

So,

$$
\begin{aligned}
L_{41}: & =t\left(1-\sum_{j=1}^{m-2} \beta_{j}\right) \sum_{j=i}^{m-2} \alpha_{j}\left(\xi_{j}-s\right) \\
& \geq\left(1-\sum_{j=1}^{m-2} \beta_{j}\right) \sum_{j=1}^{m-2} \alpha_{j} \xi_{j}(t-s)-\left(1-\sum_{j=1}^{m-2} \beta_{j}\right) \sum_{j=1}^{i-1} \alpha_{j} \xi_{j}(t-s) .
\end{aligned}
$$

Therefore

$$
\begin{aligned}
L_{43}+L_{42}+L_{41} \geq & {\left[\left(1-\sum_{j=1}^{m-2} \beta_{j}\right)\left(a+\sum_{j=1}^{m-2} \alpha_{j} \xi_{j}\right)\right.} \\
& \left.+\left(1-\sum_{j=1}^{m-2} \alpha_{j}\right)\left(b+1-\sum_{j=1}^{m-2} \beta_{j} \xi_{j}\right)\right](t-s)=M(t-s) .
\end{aligned}
$$


According to the above discussion, we get

$$
L_{4}=L_{43}+L_{42}+L_{41}-M(t-s) \geq 0 .
$$

Lemma 3.4. Suppose $\left(H_{1}\right)$ holds. Then $g_{i}(t, s) \leq 0(0 \leq i \leq n-1)$, where $g_{i}(t, s)$ is the Green's function for the problem

$$
\left\{\begin{array}{l}
x^{\prime \prime}(t)=0, \quad 0 \leq t \leq 1, \\
x(0)-a_{i} x^{\prime}(0)=\sum_{j=1}^{m-2} \alpha_{i j} x\left(\xi_{j}\right), \quad x(1)+b_{i} x^{\prime}(1)=\sum_{j=1}^{m-2} \beta_{i j} x\left(\xi_{j}\right) .
\end{array}\right.
$$

Proof. It is easy to see that $g_{i}(t, s) \leq 0$ by using Lemma 3.3.

Let $G_{1}(t, s)=g_{n-2}(t, s)$, then for $2 \leq j \leq n-1$ we recursively define

$$
G_{j}(t, s)=\int_{0}^{1} g_{n-j-1}(t, r) G_{j-1}(r, s) d r .
$$

Lemma 3.5. Suppose $\left(H_{1}\right)$ holds. If $f(t) \in C[0,1]$, then the boundary value problem

$$
\left\{\begin{array}{l}
u^{(2 l)}(t)=f(t), \quad 0 \leq t \leq 1, \\
u^{(2 i)}(0)-a_{n-l+i-1} u^{(2 i+1)}(0)=\sum_{j=1}^{m-2} \alpha_{n-l+i-1, j} u^{(2 i)}\left(\xi_{j}\right), \\
u^{(2 i)}(1)+b_{n-l+i-1} u^{(2 i+1)}(1)=\sum_{j=1}^{m-2} \beta_{n-l+i-1, j} u^{(2 i)}\left(\xi_{j}\right), \quad 0 \leq i \leq l-1,
\end{array}\right.
$$

has a unique solution for each $1 \leq l \leq n-1, G_{l}(t, s)$ is the associated Green's function for the boundary value problem (6).

Proof. We prove the result by using induction. Obviously, the result holds by using Lemma 3.2 for $l=1$.

We assume that the result holds for $l-1$. Now we consider the case for $l$. Let $u^{\prime \prime}(t)=v(t)$, then (6) is equivalent to

$$
\left\{\begin{array}{l}
u^{\prime \prime}(t)=v(t), \quad 0 \leq t \leq 1 \\
u(0)-a_{n-l-1} u^{\prime}(0)=\sum_{j=1}^{m-2} \alpha_{n-l-1, j} u\left(\xi_{j}\right) \\
u(1)+b_{n-l-1} u^{\prime}(1)=\sum_{j=1}^{m-2} \beta_{n-l-1, j} u\left(\xi_{j}\right)
\end{array}\right.
$$

and

$$
\left\{\begin{array}{l}
v^{(2(l-1))}(t)=f(t), \quad 0 \leq t \leq 1 \\
v^{(2 i)}(0)-a_{n-l+i} v^{(2 i+1)}(0)=\sum_{j=1}^{m-2} \alpha_{n-l+i, j} v^{(2 i)}\left(\xi_{j}\right), \\
v^{(2 i)}(1)+b_{n-l+i} v^{(2 i+1)}(1)=\sum_{j=1}^{m-2} \beta_{n-l+i, j} v^{(2 i)}\left(\xi_{j}\right), \quad 0 \leq i \leq l-2 .
\end{array}\right.
$$


Lemma 3.2 implies that (7) has a unique solution $u(t)=\int_{0}^{1} g_{n-l-1}(t, r) v(r) d r$, and (8) has also a unique solution $v(t)=\int_{0}^{1} G_{l-1}(t, s) f(s) d s$ by the inductive hypothesis. Thus (6) has a unique solution

$$
\begin{aligned}
u(t) & =\int_{0}^{1} g_{n-l-1}(t, r) \int_{0}^{1} G_{l-1}(r, s) f(s) d s d r \\
& =\int_{0}^{1}\left(\int_{0}^{1} g_{n-l-1}(t, r) G_{l-1}(r, s) d r\right) f(s) d s \\
& =\int_{0}^{1} G_{l}(t, s) f(s) d s .
\end{aligned}
$$

Therefore the result holds for $l$. The proof of Lemma 3.5 is now completed.

For each $1 \leq l \leq n-1$, we define $A_{l}: C[0,1] \rightarrow C[0,1]$ by

$$
A_{l} v(t)=\int_{0}^{1} G_{l}(t, \tau) v(\tau) d \tau
$$

With the use of Lemma 3.5, for each $1 \leq l \leq n-1$ we have

$$
\left\{\begin{array}{l}
\left(A_{l} v\right)^{(2 l)}(t)=v(t), \quad 0 \leq t \leq 1, \\
\left(A_{l} v\right)^{(2 i)}(0)-a_{n-l+i-1}\left(A_{l} v\right)^{(2 i+1)}(0)=\sum_{j=1}^{m-2} \alpha_{n-l+i-1, j}\left(A_{l} v\right)^{(2 i)}\left(\xi_{j}\right), \\
\left(A_{l} v\right)^{(2 i)}(1)+b_{n-l+i-1}\left(A_{l} v\right)^{(2 i+1)}(1)=\sum_{j=1}^{m-2} \beta_{n-l+i-1, j}\left(A_{l} v\right)^{(2 i)}\left(\xi_{j}\right), \\
0 \leq i \leq l-1 .
\end{array}\right.
$$

Therefore (4) has a solution if and only if the boundary value problem

$$
\left\{\begin{array}{l}
v^{\prime \prime}(t)=f\left(t, A_{n-1} v(t), A_{n-2} v(t), \ldots, A_{1} v(t), v(t)\right), \quad 0 \leq t \leq 1, \\
v(0)-a_{n-1} v^{\prime}(0)=\sum_{j=1}^{m-2} \alpha_{n-1, j} v\left(\xi_{j}\right), v(1)+b_{n-1} v^{\prime}(1)=\sum_{j=1}^{m-2} \beta_{n-1, j} v\left(\xi_{j}\right),
\end{array}\right.
$$

has a solution. If $x$ is a solution of (4), then $v=x^{(2(n-1))}$ is a solution of (9). Conversely, if $v$ is a solution of (9), then $x=A_{n-1} v$ is a solution of (4).

Define $A: C[0,1] \rightarrow C[0,1]$ by

$$
A v(t)=\int_{0}^{1} g_{n-1}(t, s) f\left(s, A_{n-1} v(s), A_{n-2} v(s), \ldots, A_{1} v(s), v(s)\right) d s .
$$

It now follows that there exists a solution of BVP (4) if and only if there exists a fixed point of $A$. Moreover, the relationship between a solution of BVP (4) and a fixed point of $A$ is given by $x=A_{n-1} v(t)$ or equivalently, by $x^{(2(n-1))}=v$. 
Note that $x$ is a positive solution of $(4)$ if and only if $(-1)^{n-1} x^{(2(n-1))}=$ $(-1)^{n-1} v$ is positive, where $v$ is the corresponding fixed point of $A$.

For each $0 \leq t \leq 1,0 \leq i \leq n-1$, there is only a finite number of points $s$ such that $g_{i}(t, s)=0$. Let

$$
M_{i}=\max _{0 \leq t \leq 1} \int_{0}^{1}\left|g_{i}(t, s)\right| d s, \quad m_{i}=\min _{\delta \leq t \leq 1-\delta} \int_{\delta}^{1-\delta}\left|g_{i}(t, s)\right| d s,
$$

where $0<\delta<\min \left\{\xi_{1}, 1-\xi_{m-2}\right\}$. It is obvious that $M_{i}>m_{i}>0$.

Let $X=C[0,1]$ with the maximum norm $\|x\|=\max _{0 \leq t \leq 1}|x(t)|$ and define the cone $P \subset X$ by

$$
P=\left\{x \in X:(-1)^{n-1} x(t) \geq 0,(-1)^{n-1} x \text { is concave on }[0,1]\right\} .
$$

Let $\alpha: P \rightarrow[0, \infty)$ be a nonnegative continuous concave functional

$$
\alpha(x)=\min _{\delta \leq t \leq 1-\delta}(-1)^{n-1} x(t) \quad \text { for } \quad x \in P .
$$

We now present our main result.

Theorem 3.1. Suppose $\left(H_{1}\right)$ and $\left(H_{2}\right)$ hold. In addition let there exist nonnegative numbers $a, b$, and $c$ such that $0<a<b \leq \min \left\{\delta, m_{n-1} / M_{n-1}\right\} c$ and $f\left(t, u_{n-1}, u_{n-2}, \ldots, u_{1}, u_{0}\right)$ satisfy the following growth conditions:

$\left(H_{3}\right)(-1)^{n} f\left(t, u_{n-1}, \ldots, u_{0}\right)<a / M_{n-1}$ for $\left(t,\left|u_{n-1}\right|,\left|u_{n-2}\right|, \ldots,\left|u_{0}\right|\right) \in[0,1] \times$ $\prod_{j=n-1}^{1}\left[0, \prod_{i=2}^{j+1} M_{n-i} a\right] \times[0, a]$

$\left(H_{4}\right)(-1)^{n} f\left(t, u_{n-1}, \ldots, u_{0}\right)<c / M_{n-1}$ for $\left(t,\left|u_{n-1}\right|,\left|u_{n-2}\right|, \ldots,\left|u_{0}\right|\right) \in[0,1] \times$ $\prod_{j=n-1}^{1}\left[0, \prod_{i=2}^{j+1} M_{n-i} c\right] \times[0, c]$

$\left(H_{5}\right)(-1)^{n} f\left(t, u_{n-1}, \ldots, u_{0}\right) \geq b / m_{n-1}$ for $\left(t,\left|u_{n-1}\right|,\left|u_{n-2}\right|, \ldots,\left|u_{0}\right|\right) \in[\delta, 1-$ $\delta] \times \prod_{j=n-1}^{1}\left[\prod_{i=2}^{j+1} m_{n-i} b, \prod_{i=2}^{j+1} M_{n-i} b / \delta\right] \times[b, b / \delta]$.

Then the boundary value problem (4) has at least three positive solutions $x_{1}$, $x_{2}$ and $x_{3}$ such that

$$
\left\|x_{1}^{(2(n-1))}\right\|<a, \quad b<\min _{\delta \leq t \leq 1-\delta}(-1)^{n-1} x_{2}^{(2(n-1))}(t)
$$

and

$$
\left\|x_{3}^{(2(n-1))}\right\|>a \quad \text { with } \min _{\delta \leq t \leq 1-\delta}(-1)^{n-1} x_{3}^{(2(n-1))}(t)<b .
$$

Proof. First we show that $A: P \rightarrow P$. Let $x \in P$. Then $(-1)^{n-1} A x(t) \geq 0$. Moreover,

$$
(-1)^{n-1}(A x)^{\prime \prime}(t)=(-1)^{n-1} f\left(t, A_{n-1} x(t), A_{n-2} x(t), \ldots, A_{1} x(t), x(t)\right)<0 .
$$

This concavity implies that $A: P \rightarrow P$. Also, it is easy to see that the operator $A$ is completely continuous.

Choose $x \in \bar{P}_{c}$, then $\|x\| \leq c$. Note that

$$
\left\|A_{j} x\right\|=\max _{t \in[0,1]}\left|\int_{0}^{1} G_{j}(t, s) x(s) d s\right| \leq \prod_{i=2}^{j+1} M_{n-i}\|x\| \leq \prod_{i=2}^{j+1} M_{n-i} c .
$$


Thus, according to assumption $\left(H_{4}\right)$, we have

$$
\begin{aligned}
\|A x\| & =\max _{0 \leq t \leq 1}|A x(t)| \\
& =\max _{0 \leq t \leq 1}\left\{\int_{0}^{1}\left|g_{n-1}(t, s) f\left(s, A_{n-1} x(s), A_{n-2} x(s), \ldots, A_{1} x(s), x(s)\right)\right| d s\right\} \\
& \leq \frac{c}{M_{n-1}} \max _{0 \leq t \leq 1}\left\{\int_{0}^{1}\left|g_{n-1}(t, s)\right| d s\right\} \\
& =c .
\end{aligned}
$$

Therefore $A: \bar{P}_{c} \rightarrow \bar{P}_{c}$.

Analogously, assumption $\left(H_{3}\right)$ implies that Condition $(\mathrm{C} 2)$ of the LeggettWilliams Fixed Point Theorem is satisfied.

We now show that condition $(\mathrm{C} 1)$ is satisfied. Note that for $0 \leq t \leq 1$,

$$
x(t)=(-1)^{n-1} \frac{b}{\delta} \in P\left(\alpha, b, \frac{b}{\delta}\right) \quad \text { and } \quad \alpha(x)=\frac{b}{\delta}>b .
$$

Thus

$$
\left\{x \in P\left(\alpha, b, \frac{b}{\delta}\right) \mid \alpha(x)>b\right\} \neq \varnothing .
$$

Also, if $x \in P\left(\alpha, b, \frac{b}{\delta}\right)$, then $\alpha(x)=\min _{\delta \leq t \leq 1-\delta}(-1)^{n-1} x(t) \geq b$ for each $\delta \leq t \leq$ $1-\delta$, so $(-1)^{n-1} x(t) \geq b, \delta \leq t \leq 1-\delta$ and therefore

$$
\begin{aligned}
(-1)^{n-2} A_{1} x(t) & =\int_{0}^{1}-G_{1}(t, s)(-1)^{n-1} x(s) d s \\
& \geq \int_{\delta}^{1-\delta}-G_{1}(t, s)(-1)^{n-1} x(s) d s \\
& \geq b \int_{\delta}^{1-\delta}\left|G_{1}(t, s)\right| d s \geq b m_{n-2} .
\end{aligned}
$$

Inductively, we have

$$
(-1)^{n-1-j} A_{j} x(t) \geq \prod_{i=2}^{j+1} m_{n-j} b, \quad \delta \leq t \leq 1-\delta, \quad 1 \leq j \leq n-1,
$$

and it is easy to see that

$$
\left|A_{j} x(t)\right| \leq \prod_{i=2}^{j+1} M_{n-j} \frac{b}{\delta}
$$


Applying now assumption $\left(H_{5}\right)$, we obtain

$$
(-1)^{n} f\left(t, A_{n-1} x(t), A_{n-2} x(t), \ldots, A_{1} x(t), x(t)\right) \geq \frac{b}{m_{n-1}}, \quad \delta \leq t \leq 1-\delta .
$$

So,

$$
\begin{aligned}
& \alpha(A x)=\min _{\delta \leq t \leq 1-\delta}(-1)^{n-1} A x(t) \\
& =\min _{\delta \leq t \leq 1-\delta}\left\{\int_{0}^{1}-g_{n-1}(t, s)(-1)^{n} f\left(s, A_{n-1} x(s), A_{n-2} x(s), \ldots, A_{1} x(s), x(s)\right) d s\right\} \\
& >\min _{\delta \leq t \leq 1-\delta}\left\{\int_{\delta}^{1-\delta}-g_{n-1}(t, s)(-1)^{n} f\left(s, A_{n-1} x(s), A_{n-2} x(s), \ldots, A_{1} x(s), x(s)\right) d s\right\} \\
& \geq \frac{b}{m_{n-1}} \min _{\delta \leq t \leq 1-\delta} \int_{\delta}^{1-\delta}\left|g_{n-1}(t, s)\right| d s \\
& =b .
\end{aligned}
$$

Therefore condition (C1) is satisfied.

Finally, we show that condition (C3) is also satisfied, i.e., we show that if $x \in P(\alpha, b, c)$ and $\|A x\|>d=b / \delta$, then $\alpha(A x)>b$. This follows since $A: P \rightarrow P$, in particular, since $(-1)^{n-1}(A x)$ is concave,

$$
\min _{\delta \leq t \leq 1-\delta}(-1)^{n-1} A x(t) \geq \delta\|A x\| \text {. }
$$

Thus $\alpha(A x)>\delta d=b$. Therefore condition (C3) is also satisfied. So we complete the proof.

\section{EXAMPLE}

In this section, we present an example to demonstrate the application of Theorem 3.1. Consider the boundary value problem

$$
\left\{\begin{array}{l}
x^{(4)}(t)=f\left(t, x(t), x^{\prime \prime}(t)\right), \quad 0 \leq t \leq 1 \\
x(0)-a_{0} x^{\prime}(0)=\frac{1}{2} x\left(\frac{1}{2}\right), \quad x(1)+b_{0} x^{\prime}(1)=\frac{1}{2} x\left(\frac{1}{2}\right) \\
x^{\prime \prime}(0)-a_{1} x^{(3)}(0)=\frac{1}{2} x^{\prime \prime}\left(\frac{1}{2}\right), \quad x^{\prime \prime}(1)+b_{1} x^{(3)}(1)=\frac{1}{2} x^{\prime \prime}\left(\frac{1}{2}\right)
\end{array}\right.
$$

where

$$
f(t, x, y)=\left\{\begin{array}{l}
\frac{1}{1000} \sin t+x+\frac{1}{1000} y^{3}, \quad x \in\left(-\infty, \frac{89}{256}\right], \\
\frac{1}{1000} \sin t-\frac{160000}{9}\left(x-\frac{23}{64}\right)^{2}+\frac{357}{128}+\frac{1}{1000} y^{3}, \quad x \in\left[\frac{89}{256}, \frac{23}{64}\right], \\
\frac{1}{1000} \sin t-\frac{80}{1089}\left(x-\frac{89}{64}\right)^{2}+\frac{367}{128}+\frac{1}{1000} y^{3}, \quad x \in\left[\frac{23}{64}, \frac{89}{64}\right], \\
\frac{1}{1000} \sin t+\frac{80}{1089}\left(x-\frac{89}{64}\right)^{2}+\frac{367}{128}+\frac{1}{1000} y^{3}, \quad x \in\left[\frac{89}{64}, \frac{89}{32}\right], \\
\frac{1}{1000} \sin t+\frac{838931}{278784}+\frac{1}{1000} y^{3}, \quad x \in\left[\frac{89}{32},+\infty\right) .
\end{array}\right.
$$


Let $a_{0}=\frac{3}{4}, b_{0}=\frac{1}{4}, a_{1}=\frac{1}{4}, b_{1}=\frac{3}{4}, \delta=\frac{1}{4}$, then we have

$$
\begin{aligned}
& \left|g_{0}(t, s)\right|= \begin{cases}\left(s+\frac{3}{4}\right)\left(1-\frac{1}{2} t\right), \quad 0 \leq t \leq 1, \quad 0 \leq s \leq \frac{1}{2}, \quad s \leq t \\
\frac{3}{8}(s-t)+\left(t+\frac{3}{4}\right)\left(1-\frac{1}{2} s\right), \quad 0 \leq t \leq 1,0 \leq s \leq \frac{1}{2}, \quad t \leq s \\
\frac{5}{8}(t-s)+\left(\frac{5}{4}-t\right)\left(1+\frac{1}{2} s\right), \quad 0 \leq t \leq 1, \quad \frac{1}{2} \leq s \leq 1, \quad s \leq t \\
\left(\frac{5}{4}-s\right)\left(\frac{1}{2} t+1\right), \quad 0 \leq t \leq 1, \quad \frac{1}{2} \leq s \leq 1, t \leq s .\end{cases} \\
& \left|g_{1}(t, s)\right|= \begin{cases}\left(s+\frac{1}{4}\right)\left(\frac{3}{2}-\frac{1}{2} t\right), \quad 0 \leq t \leq 1,0 \leq s \leq \frac{1}{2}, \quad s \leq t ; \\
\frac{5}{8}(s-t)+\left(t+\frac{1}{4}\right)\left(\frac{3}{2}-\frac{1}{2} s\right), & 0 \leq t \leq 1, \quad 0 \leq s \leq \frac{1}{2}, t \leq s ; \\
\frac{3}{8}(t-s)+\left(\frac{7}{4}-t\right)\left(\frac{1}{2}+\frac{1}{2} s\right), & 0 \leq t \leq 1, \quad \frac{1}{2} \leq s \leq 1, \quad s \leq t ; \\
\left(\frac{7}{4}-s\right)\left(\frac{1}{2} t+\frac{1}{2}\right), \quad 0 \leq t \leq 1, & \frac{1}{2} \leq s \leq 1, t \leq s .\end{cases}
\end{aligned}
$$

We first consider the condition $i=0$.

1) For $0 \leq t \leq \frac{1}{2}$ we have

$$
\begin{aligned}
\int_{0}^{1}\left|g_{0}(t, s)\right| d s= & \int_{0}^{t}\left|g_{0}(t, s)\right| d s+\int_{t}^{\frac{1}{2}}\left|g_{0}(t, s)\right| d s+\int_{\frac{1}{2}}^{1}\left|g_{0}(t, s)\right| d s \\
= & \int_{0}^{t}\left(s+\frac{3}{4}\right)\left(1-\frac{1}{2} t\right) d s+\int_{t}^{\frac{1}{2}}\left[\frac{3}{8}(s-t)+\left(t+\frac{3}{4}\right)\left(1-\frac{1}{2} s\right)\right] d s \\
& +\int_{\frac{1}{2}}^{1}\left(\frac{5}{4}-s\right)\left(\frac{1}{2} t+1\right) d s \\
= & \frac{5}{8}+\frac{3}{8} t-\frac{1}{2} t^{2} .
\end{aligned}
$$

2) For $\frac{1}{2} \leq t \leq 1$ we have

$$
\begin{aligned}
\int_{0}^{1}\left|g_{0}(t, s)\right| d s= & \int_{0}^{\frac{1}{2}}\left|g_{0}(t, s)\right| d s+\int_{\frac{1}{2}}^{t}\left|g_{0}(t, s)\right| d s+\int_{t}^{1}\left|g_{0}(t, s)\right| d s \\
= & \int_{0}^{\frac{1}{2}}\left(s+\frac{3}{4}\right)\left(1-\frac{1}{2} t\right) d s+\int_{\frac{1}{2}}^{t}\left[\frac{5}{8}(t-s)+\left(\frac{5}{4}-t\right)\left(1+\frac{1}{2} s\right)\right] d s \\
& +\int_{t}^{1}\left(\frac{5}{4}-s\right)\left(\frac{1}{2} t+1\right) d s \\
= & \frac{5}{8}+\frac{3}{8} t-\frac{1}{2} t^{2} .
\end{aligned}
$$


So,

$$
M_{0}=\max _{0 \leq t \leq 1} \int_{0}^{1}\left|g_{0}(t, s)\right| d s=\frac{89}{128}
$$

On the other hand,

3) For $\frac{1}{4} \leq t \leq \frac{1}{2}$ we have

$$
\begin{aligned}
\int_{\frac{1}{4}}^{\frac{3}{4}}\left|g_{0}(t, s)\right| d s= & \int_{\frac{1}{4}}^{t}\left|g_{0}(t, s)\right| d s+\int_{t}^{\frac{1}{2}}\left|g_{0}(t, s)\right| d s+\int_{\frac{1}{2}}^{\frac{3}{4}}\left|g_{0}(t, s)\right| d s \\
= & \int_{\frac{1}{4}}^{t}\left(s+\frac{3}{4}\right)\left(1-\frac{1}{2} t\right) d s+\int_{t}^{\frac{1}{2}}\left[\frac{3}{8}(s-t)+\left(t+\frac{3}{4}\right)\left(1-\frac{1}{2} s\right)\right] d s \\
& +\int_{\frac{1}{2}}^{\frac{3}{4}}\left(\frac{5}{4}-s\right)\left(\frac{1}{2} t+1\right) d s \\
= & \frac{5}{16}+\frac{7}{16} t-\frac{1}{2} t^{2} .
\end{aligned}
$$

4) For $\frac{1}{2} \leq t \leq \frac{3}{4}$ we have

$$
\begin{aligned}
\int_{\frac{1}{4}}^{\frac{3}{4}}\left|g_{0}(t, s)\right| d s= & \int_{\frac{1}{4}}^{\frac{1}{2}}\left|g_{0}(t, s)\right| d s+\int_{\frac{1}{2}}^{t}\left|g_{0}(t, s)\right| d s+\int_{t}^{\frac{3}{4}}\left|g_{0}(t, s)\right| d s \\
= & \int_{\frac{1}{4}}^{\frac{1}{2}}\left(s+\frac{3}{4}\right)\left(1-\frac{1}{2} t\right) d s+\int_{\frac{1}{2}}^{t}\left[\frac{5}{8}(t-s)+\left(\frac{5}{4}-t\right)\left(1+\frac{1}{2} s\right)\right] d s \\
& +\int_{t}^{\frac{3}{4}}\left(\frac{5}{4}-s\right)\left(\frac{1}{2} t+1\right) d s \\
= & \frac{5}{16}+\frac{7}{16} t-\frac{1}{2} t^{2} .
\end{aligned}
$$

So,

$$
m_{0}=\min _{\frac{1}{4} \leq t \leq \frac{3}{4}} \int_{\frac{1}{4}}^{\frac{3}{4}}\left|g_{0}(t, s)\right| d s=\frac{23}{64} .
$$

Next, we consider the condition $i=1$. 
5) For $0 \leq t \leq \frac{1}{2}$ we have

$$
\begin{aligned}
\int_{0}^{1}\left|g_{1}(t, s)\right| d s= & \int_{0}^{t}\left|g_{1}(t, s)\right| d s+\int_{t}^{\frac{1}{2}}\left|g_{1}(t, s)\right| d s+\int_{\frac{1}{2}}^{1}\left|g_{1}(t, s)\right| d s \\
= & \int_{0}^{t}\left(s+\frac{1}{4}\right)\left(\frac{3}{2}-\frac{1}{2} t\right) d s+\int_{t}^{\frac{1}{2}}\left[\frac{5}{8}(s-t)+\left(t+\frac{1}{4}\right)\left(\frac{3}{2}-\frac{1}{2} s\right)\right] d s \\
& +\int_{\frac{1}{2}}^{1}\left(\frac{7}{4}-s\right)\left(\frac{1}{2} t+\frac{1}{2}\right) d s \\
= & \frac{1}{2}+\frac{5}{8} t-\frac{1}{2} t^{2} .
\end{aligned}
$$

6) For $\frac{1}{2} \leq t \leq 1$ we have

$$
\begin{aligned}
\int_{0}^{1}\left|g_{1}(t, s)\right| d s= & \int_{0}^{\frac{1}{2}}\left|g_{1}(t, s)\right| d s+\int_{\frac{1}{2}}^{t}\left|g_{1}(t, s)\right| d s+\int_{t}^{1}\left|g_{1}(t, s)\right| d s \\
= & \int_{0}^{\frac{1}{2}}\left(s+\frac{1}{4}\right)\left(\frac{3}{2}-\frac{1}{2} t\right) d s+\int_{\frac{1}{2}}^{t}\left[\frac{3}{8}(t-s)+\left(\frac{7}{4}-t\right)\left(\frac{1}{2}+\frac{1}{2} s\right)\right] d s \\
& +\int_{t}^{1}\left(\frac{7}{4}-s\right)\left(\frac{1}{2} t+\frac{1}{2}\right) d s \\
= & \frac{1}{2}+\frac{5}{8} t-\frac{1}{2} t^{2} .
\end{aligned}
$$

So,

$$
M_{1}=\max _{0 \leq t \leq 1} \int_{0}^{1}\left|g_{1}(t, s)\right| d s=\frac{89}{128} .
$$

On the other hand,

7) For $\frac{1}{4} \leq t \leq \frac{1}{2}$ we have

$$
\begin{aligned}
\int_{\frac{1}{4}}^{\frac{3}{4}}\left|g_{1}(t, s)\right| d s & =\int_{\frac{1}{4}}^{t}\left|g_{1}(t, s)\right| d s+\int_{t}^{\frac{1}{2}}\left|g_{1}(t, s)\right| d s+\int_{\frac{1}{2}}^{\frac{3}{4}}\left|g_{1}(t, s)\right| d s \\
& =\int_{\frac{1}{4}}^{t}\left(s+\frac{1}{4}\right)\left(\frac{3}{2}-\frac{1}{2} t\right) d s+\int_{t}^{\frac{1}{2}}\left[\frac{5}{8}(s-t)+\left(t+\frac{1}{4}\right)\left(\frac{3}{2}-\frac{1}{2} s\right)\right] d s
\end{aligned}
$$




$$
\begin{aligned}
& +\int_{\frac{1}{2}}^{\frac{3}{4}}\left(\frac{7}{4}-s\right)\left(\frac{1}{2} t+\frac{1}{2}\right) d s \\
= & \frac{1}{4}+\frac{9}{16} t-\frac{1}{2} t^{2} .
\end{aligned}
$$

8) For $\frac{1}{2} \leq t \leq \frac{3}{4}$ we have

$$
\begin{aligned}
\int_{\frac{1}{4}}^{\frac{3}{4}}\left|g_{1}(t, s)\right| d s= & \int_{\frac{1}{4}}^{\frac{1}{2}}\left|g_{1}(t, s)\right| d s+\int_{\frac{1}{2}}^{t}\left|g_{1}(t, s)\right| d s+\int_{t}^{\frac{3}{4}}\left|g_{1}(t, s)\right| d s \\
= & \int_{\frac{1}{4}}^{\frac{1}{2}}\left(s+\frac{1}{4}\right)\left(\frac{3}{2}-\frac{1}{2} t\right) d s+\int_{\frac{1}{2}}^{t}\left[\frac{3}{8}(t-s)+\left(\frac{7}{4}-t\right)\left(\frac{1}{2}+\frac{1}{2} s\right)\right] d s \\
& +\int_{t}^{\frac{3}{4}}\left(\frac{7}{4}-s\right)\left(\frac{1}{2} t+\frac{1}{2}\right) d s \\
= & \frac{1}{4}+\frac{9}{16} t-\frac{1}{2} t^{2} .
\end{aligned}
$$

So,

$$
m_{1}=\min _{\frac{1}{4} \leq t \leq \frac{3}{4}} \int_{\frac{1}{4}}^{\frac{3}{4}}\left|g_{1}(t, s)\right| d s=\frac{23}{64} .
$$

Let $a=\frac{1}{2}, b=1, c=2$, then

$$
\begin{array}{rlll}
f(t, x, y)<a / M_{1}=\frac{64}{89} & \text { for } & (t,|x|,|y|) \in[0,1] \times\left[0, \frac{89}{256}\right] \times\left[0, \frac{1}{2}\right], \\
f(t, x, y)<c / M_{1}=\frac{256}{89} & \text { for } & (t,|x|,|y|) \in[0,1] \times\left[0, \frac{89}{64}\right] \times[0,2], \\
f(t, x, y) \geq b / m_{1}=\frac{64}{23} & \text { for } & (t,|x|,|y|) \in\left[\frac{1}{4}, \frac{3}{4}\right] \times\left[\frac{23}{64}, \frac{89}{32}\right] \times[1,4] .
\end{array}
$$

By Theorem 3.1, problem (4.1) has at least three positive solutions.

\section{ACKNOWLEDGMENTS}

This research was supported by the National Natural Science Foundation of China (No. 10671012).

\section{REFERENCES}

1. R. W. Leggett and L. R. Williams, Multiple positive fixed points of nonlinear operators on ordered Banach spaces. Indiana Univ. Math. J. 28(1979), No. 4, 673-688.

2. V. A. IL'in and E. I. Moiseev, A nonlocal boundary value problem of the second kind for the Sturm-Liouville operator. (Russian) Differentsial'nye Uravneniya 23(1987), No. 8, 1422-1431. 
3. V. A. IL'In and E. I. Moiseev, A nonlocal boundary value problem of the first kind for the Sturm-Liouville operator in differential and difference interpretations. (Russian) Differentsial'nye Uravneniya 23(1987), No. 7, 1198-1207.

4. W. Feng and J. R. L. WebB, Solvability of $m$-point boundary value problems with nonlinear growth. J. Math. Anal. Appl. 212(1997), No. 2, 467-480.

5. W. Feng, On an $M$-point boundary value problem. Proceedings of the Second World Congress of Nonlinear Analysts, Part 8 (Athens, 1996). Nonlinear Anal. 30(1997), No. 8, 5369-5374.

6. C. P. Gupta, Solvability of a three-point nonlinear boundary value problem for a second order ordinary differential equation. J. Math. Anal. Appl. 168(1992), No. 2, 540-551.

7. C. P. Gupta, A sharper condition for the solvability of a three-point second order boundary value problem. J. Math. Anal. Appl. 205(1997), No. 2, 586-597.

8. C. P. Gupta, A generalized multi-point boundary value problem for second order ordinary differential equations. Differential equations and computational simulations, II (Mississippi State, MS, 1995). Appl. Math. Comput. 89(1998), No. 1-3, 133-146.

9. J. M. Davis, P. W. Eloe, and J. Henderson, Triple positive solutions and dependence on higher order derivatives. J. Math. Anal. Appl. 237(1999), No. 2, 710-720.

10. J. M. Davis, J. Henderson, and P. J. Y. Wong, General Lidstone problems: multiplicity and symmetry of solutions. J. Math. Anal. Appl. 251(2000), No. 2, 527-548.

11. Y. GuO, W. Ge, and Y. GaO, Twin positive solutions for higher order $m$-point boundary value problems with sign changing nonlinearities. Appl. Math. Comput. 146(2003), No. 2-3, 299-311.

12. Y. Guo, X. LiU, and J. QIU, Three positive solutions for higher order $m$-point boundary value problems. J. Math. Anal. Appl. 289(2004), No. 2, 545-553.

(Received 12.10.2004; revised 10.10.2006)

Authors' addresses:

Youyu Wang

Department of Mathematics

Tianjin University of Finance and Economics

Tianjin 300222

P.R. China

E-mail: wang_youyu@163.com

Weigao Ge

Department of Mathematics

Beijing Institute of Technology

Beijing 100081

P.R. China

E-mail: gew@bit.edu.cn 